\title{
Selective Distortion of Body Image by Asynchronous
}

\section{Visuotactile Stimulation}

\author{
Daniel Perez-Marcos ${ }^{1,2^{*}}$, Matteo Martini ${ }^{1,2}$, Christina T. Fuentes ${ }^{3}$, Anna Bellido ${ }^{2}$, Patrick \\ Haggard $^{3}$, Maria V. Sanchez-Vives ${ }^{1,2,4}$ \\ ${ }^{1}$ Institut d'Investigacions Biomèdiques August Pi i Sunyer (IDIBAPS), Barcelona, Spain \\ ${ }^{2}$ EVENT-Lab, Facultat de Psicologia, Universitat de Barcelona, Barcelona, Spain \\ ${ }^{3}$ Institute of Cognitive Neuroscience, University College London, London, UK \\ ${ }^{4}$ ICREA, Barcelona, Spain
}

*Corresponding author:

E-mail : daniel.perez.marcos@gmail.com (DPM) 


\section{Abstract}

5

6

\section{Selective Distortion of Body Image by Asynchronous}

\section{Visuotactile Stimulation}

In the rubber hand illusion (RHI), a rubber hand is felt as being part of one's body. This

illusion is evoked by providing synchronous visuotactile stimulation to the fake and real hands.

Asynchronous visuotactile stimulation is known not to produce such an illusion of ownership,

being commonly used as the control condition. Here we explored the impact of synchronous and asynchronous visuotactile stimulation on the body image. We combined the induction of the RHI with a quantitative test for the internal representation of body metrics (i.e., the positions of key fiducial points on the body relative to each other). We found a significant recalibration of the upper/lower arm lengths following asynchronous visuotactile stimulation. In particular, we observed a selective elongation of the lower arm, a distortion typical of deafferentation.

Conversely, synchronous visuotactile stimulation did not alter the estimation of the arm segments' length. Our findings are consistent with a dynamic internal representation of body image that is continuously updated based on incoming multisensory information. Furthermore, the use of asynchronous multisensory stimulation as a neutral condition should be reconsidered since it introduces changes in the body image.

Keywords: rubber hand illusion, body image, visuotactile stimulation, multisensory correlations, body ownership, body representation. 


\section{Introduction}

The perceptual representation of one's own body (i.e., body image) can be altered by several clinical conditions (Flannery \& Faria, 1999) and by experimental manipulations (Botvinick \& Cohen, 1998), inducing illusory body experiences. For example, in the case of clinical conditions, the amputation of a limb often leads to the perception of a phantom limb, with the person often feeling pain in the area from which the limb has been amputated. This phantom limb becomes progressively shorter, a phenomenon called "telescoping" (Ramachandran \& Hirstein, 1998). In contrast, when a body part is deafferented (deprived of sensory input), for example by local anaesthesia, the feeling of an increased size of that body part often occurs (Gandevia \& Phegan, 1999; Paqueron et al., 2003). Such an effect has also been observed in patients with spinal cord injury that perceived their torso and limbs elongated (Fuentes, Longo, \& Haggard, 2013). In the case of experimental manipulations, illusory body experiences can be artificially induced by providing altered multisensory inputs to the brain. In the well-known rubber hand illusion (RHI), both a fake hand and the real hand receive synchronous tactile stimulation while the real hand is kept out of view. Under these conditions, participants feel on their hand the stimulation that they see on the fake one, leading to an illusion of "owning" the fake hand (ownership), together with a feeling of touch on the fake hand (referral of touch) (Botvinick \& Cohen, 1998; Slater, Perez-Marcos, Ehrsson, \& Sanchez-Vives, 2008). Such hand illusions can be induced in patients with amputations and can also extend to other body parts. For example, Ehrsson and colleagues artificially reversed the telescoping phenomenon (i.e., the perceived shrinkage of the phantom limb) in upper limb amputees by means of the RHI, with patients feeling the "owned" hand in the original spatial location where the intact hand used to be, not in the stump (Ehrsson et al., 2008; Schmalzl et al., 2011). The same authors used a full-body illusion to induce the sensation of a telescoped limb in healthy individuals (Schmalzl \& Ehrsson, 2011). A similar telescoping effect can be induced by simultaneous vibration of 
antagonistic muscles, which generates a proprioceptive conflict leading to the perception of body part shrinkage (Longo, Kammers, Gomi, Tsakiris, \& Haggard, 2009). By means of congruent visuotactile stimulation, a virtual arm could be felt-as-own and then elongated up to three times while the illusion of ownership hardly decayed (Kilteni, Normand, Sanchez-Vives, $\&$ Slater, 2012). These studies show that our body image can be easily manipulated by illusory body experiences and that these illusory experiences may prove useful in clinical settings. While most RHI experiments use synchronous stimulation of the fake and the real hands, it is not yet known whether and how asynchronous stimulation (often used as the control) of the fake and the real hands may affect body image. A hint that asynchronous stimulation may affect body image comes from a set of rare sensations of "experiencing the hand being less vivid than normal", probably created by the intersensory conflict in the asynchronous condition and described under the umbrella term "deafference" (Longo, Schüür, Kammers, Tsakiris, \& Haggard, 2008). This sensation has been reported in healthy participants during asynchronous visuotactile stimulation (Longo et al., 2008), and that would be similar to that reported in amputees with body image alterations (Gandevia \& Phegan, 1999; Paqueron et al., 2003). To answer the posted question, we carried out two experiments that evaluated whether synchronous or asynchronous visuotactile stimulation of a fake and the real hands results in distortions of the body image. More specifically, we evaluated the differences in the perceived size of different body parts (i.e., body metrics) using the Body Image Task (BIT) (Fuentes et al., 2013) before and after the RHI. We hypothesized that illusory body experiences induced by synchronous and asynchronous visuotactile stimulation would differentially distort the body image. This new quantitative experimental approach might provide new insights into the mechanisms underlying illusory body experiences. 


\section{$1 \quad 2$ Methods}

\section{2.1 Participants}

Twenty healthy participants (10 females, 10 males; mean $\pm S D$ age: $19.9 \pm 2.3$ years) were recruited by e-mail advertising on the campus for the first experiment. Another group of 20 healthy participants ( 10 females, 10 males; age: $22.3 \pm 2.1$ years) was equally recruited for the second experiment. All participants had normal or corrected-to-normal vision and reported no history of neurological or psychological disorders and were not taking any psychotropic medication at the time of the study. Three subjects from each group were excluded from further analysis because, after outlier exclusion, no trials remained available for at least one of the limbs. Therefore, the final groups contained data from 17 subjects each. All participants were right-handed according to the Edinburgh Handedness Inventory (score>40) (Oldfield, 1971). Upon arrival at the laboratory, they were asked to read and sign a consent form. The experiment was carried out in accordance with the regulations of the Comite Ético de Investigación Clínica de la Corporación Sanitaria Hospital Clínic de Barcelona. All participants were paid $5 €$ for their participation.

\subsection{Experimental Design}

To determine whether synchronous and asynchronous visuotactile stimulation result in distortions of the body image, we carried out two experiments following the same procedure. In both experiments, participants sat comfortably in front of a desk. The real hands rested palm down on a table, the latter being covered with a black cloth. Real arms and hands were kept out of view, hidden behind a screen. Two in-white-silk rubber arms were placed face down in front of the participant in place of the real ones (Figure 1). The gap between the rubber arms and the participant's body was covered, assuring body continuity (Perez-Marcos, Sanchez-Vives, \& Slater, 2011). The horizontal distance between the real and the corresponding fake hands was 20 $\mathrm{cm}$ (middle finger-to-middle finger distance), with the rubber arms positioned closer to the body midline. Care was taken to place the rubber arms so that they appeared to have the same length 
as the real arms. The two experiments were carried out in a dim room. The only light came from

2 the ceiling, which illuminated both rubber arms equally and without shadows.

First (main) experiment. Visuotactile stimulation consisted of simultaneous tactile

4 stimulation by the experimenter's forefingers of both the real and the rubber hands, tapping and

5 stroking the hands in a synchronous ("S" condition) or asynchronous ("A" condition) fashion.

6 In the synchronous condition, participants felt the tactile stimulation on their real hand (left or

7 right, depending on the participant) while seeing the tactile stimulation only on the rubber hand

8 of the same side, coincident in location on the hand and in time. In the asynchronous condition,

9 the stimulation on the rubber and the real hands was not coincident in time and location, i.e.,

10 the stimulation site was mismatched on purpose, aiming for example at the little finger of the

11 real hand while aiming at the index finger of the rubber hand. Visuotactile stimulation started

12 either with the synchronous or the asynchronous condition (counterbalanced) on the left or right

13 hand (counterbalanced) for $20 \mathrm{~s}$, time enough to elicit the illusion (Ehrsson, Holmes, \&

14 Passingham, 2005; Lloyd, 2007). Then, the stimulation side was changed, and the opposite stimulation (asynchronous or synchronous) continued for $20 \mathrm{~s}$ on the other hand. Alternating stimulation has been effectively used by Tsakiris and colleagues (Tsakiris \& Haggard, 2005), where they stroked one finger synchronously and the other one asynchronously. In this study, the rationale behind the alternating stimulation pattern was to induce and maintain both conditions (synchronous and asynchronous) simultaneously. Doing the visuotactile stimulation alternatively on both hands had a number of advantages: it allowed the pre-test/post-test to be carried out for both conditions at the same time, enabling the fairest comparison and avoiding possible intra- and inter-session carry-over effects due to the single stimulation of one side of the body. This alternating stimulation procedure was repeated twice in a continuous sequence to reinforce the illusion of ownership (or not), as we feared that the illusion could be reduced over time due to the alternation. Overall, each hand received $40 \mathrm{~s}$ of visuotactile stimulation in total. 
1 Participants were instructed to pay attention to the rubber hand being stimulated, observing the

2 tactile stimulation performed by the experimenter.

3

Second (control) experiment. A previous study suggests that the mere vision of a fake body part from a first-person perspective may induce the feeling of ownership of the fake body part (Maselli \& Slater, 2013) and that the proprioceptive effects may gradually rise with stimulation time (Botvinick \& Cohen, 1998; Rohde, Di Luca, \& Ernst, 2009). Therefore, a control experiment was carried out with a different group of participants in order to rule out the fact that only the vision of the rubber hand per se, without any tactile stimulation (Observational condition: "O"), or synchronous visuotactile stimulation for a long time (Long-lasting Synchronous condition: "LS”) may affect body image. In this second experiment, two rubber arms were placed in front of the participant. Following a similar procedure to the one described for the first experiment, this experiment started with one of the two conditions (O or LS, counterbalanced) in the left or right hand (counterbalanced), with the only difference that the stimulation (or just observation) time lasted for $60 \mathrm{~s}$. Then the other condition was applied for $60 \mathrm{~s}$ to the other hand. This procedure was repeated twice, each hand being stimulated (or just observed) for a total of $120 \mathrm{~s}$.

\subsection{The Body Image Task (BIT)}

Before and immediately after the RHI experiment, participants completed the BIT (Fuentes et al., 2013). The BIT allows for quantification of the perceived size of body parts within one's body image. Compared to classical behavioural measurements of perceived hand position (such as proprioceptive drift), the BIT tackles different aspects of the body representation and seems to be specially sensitive to changes in the body metrics (Fuentes et al., 2013).

Participants sat about $70 \mathrm{~cm}$ in front of a PC with a screen resolution of $1024 \times 768$ pixels. The experimenter explained the participants that they would see the outline of a head near the top of the screen. They were instructed to imagine that they were looking at a mirrored image of 
themselves standing with their arms at their sides; the experimenter modelled this position by

2 standing with his legs slightly apart and his arms straight at sides with the palms facing the

3 front. Participants were told to mentally scale the image of their body to the size of the head

4 displayed on the screen. The BIT started with the name of a body part displayed on the screen

5 for $3 \mathrm{~s}$, followed by the head displayed at one of four random positions on the screen.

6 Participants used a mouse to click on the screen where, relative to the displayed head, they

7 thought the specified body part would be located on a corresponding picture of themselves.

8 Participants were given $3 \mathrm{~s}$ to respond before the next trial began. They identified the location of

912 body parts: left shoulder, right shoulder, left elbow, right elbow, left hand, right hand, left

10 hip, right hip, left knee, right knee, left foot, and right foot. Each body part was repeated four times per test in a pseudo-randomized order, making a total of 48 trials. Participants completed a three-trial practice of the BIT before starting the pre-RHI assessment.

During both pre- and post-RHI BIT, participants' arms and hands remained hidden from their view. In the post-RHI test, just after finishing the visuotactile stimulation, the rubber hands remained visible to the participant. During the entire experiment, participants remained seated and performed the BIT with their right hand.

For both experiments, perceived body part positions in the BIT were transformed into a common space using a two-point registration procedure, which involved registering sets of coordinates by aligning two of the corresponding landmarks (Bookstein, Colchester, \& Hawkes, 1991), allowing for comparison of the relative lengths of different body parts. We were interested in the perceived lengths of upper and lower arms and legs before and after the RHI. Therefore, perceived lengths of upper and lower arms and legs were normalized by dividing the perceived length of each part of the arm or leg by the perceived length of the whole arm or leg, separately for each experimental condition and time point (i.e., before and after the RHI). This normalization prevented possible effects due to different drawing scales within subjects. 


\section{$1 \quad 2.4$ Questionnaire}

2

3

After completing the post-RHI BIT, participants filled in a 4-item questionnaire to assess the sense of body ownership (Q1 and Q2) and referral of touch (Q3 and Q4; only in those conditions with tactile stimulation) of the rubber hand (Botvinick \& Cohen, 1998). Each question was scored according to a 7-point Likert Scale, 1 meaning 'totally disagree' and 7 'totally agree'. The questions were displayed in a different random order to each participant. The questions were presented in Spanish but here they have been translated to English (see Supplementary Material for the Spanish version of the questionnaire):

Q1. "During the experiment there were times when I felt as if the rubber left hand was my own left hand".

Q2. "During the experiment there were times when I felt as if the rubber right hand was my own right hand".

Q3. "Regarding the left hand, there were times when it seemed as if I were feeling the touch in the location of the left rubber hand".

Q4. "Regarding the right hand, there were times when it seemed as if I were feeling the touch in the location of the right rubber hand".

\subsection{Statistical Analysis}

Analyses were done with Statistica software (version 8.0, Stat-Soft Inc.). Responses in the BIT that were missing or reversed (i.e., left and right sides confounded) were excluded from the analysis (zero missing and 3.7\% reversed responses were excluded in the first experiment; zero and $3.5 \%$ in the second experiment). Outliers were considered values beyond a participant's median $\pm 2 * M A D$ (median absolute deviation); they were also excluded from the analysis $(22.8 \%$ excluded in the first experiment; $22.2 \%$ in the second experiment).

Variables from four different body parts (upper and lower arms, and upper and lower legs) were calculated and coded according to the type of stimulation ( $\mathrm{S}$ vs. A; O vs. LS). For all 
variables, normality was checked via Kolmogorov-Smirnov test. Then, for each experiment, a

$22 \times 2$ repeated measures ANOVA with factors "Time" (pre-RHI vs. post-RHI) and "Type of

3 Stimulation" ("ToS": S vs. A in the first experiment; O vs. LS in the second experiment) was

4 carried out for each variable. Newman-Keuls post-hoc test was used for single comparisons.

Scores for the questions referring to body ownership and referral of touch were compared separately for the first and the second experiments using a Wilcoxon matched paired-test. The level of significance was set at $p \leq .05$.

\section{Results}

\section{Body ownership and referral of touch}

In the first experiment, the RHI was successfully induced by applying synchronous visuotactile stimulation. In the synchronous (S) condition, participants scored significantly higher in body ownership and referral of touch than they did in the asynchronous (A) condition (Figure 2; Body ownership (Q1-Q2): $Z=3.52, p=.0004$; Referral of touch (Q3-Q4): $Z=3.41$, $p=.0006)$.

In the second experiment, participants scored significantly higher in body ownership in the long-lasting synchronous (LS) condition than in the observational (O) condition (Figure 3; $Z$ $=3.23, p=.0012$ ). Participants also reported high levels of referral of touch in the LS condition, similar to the $\mathrm{S}$ condition of the first experiment. The results from both experiments indicate that synchronous visuotactile stimulation induced a stronger feeling of ownership and referral of touch of the fake hand than the asynchronous visuotactile and the observational conditions did.

\section{Body metrics (BIT)}

Participants completed the BIT before (pre) and after (post) going through the RHI protocol in both experiments. Tables 1 and 2 show the perceived body part lengths for the first and the second experiment, respectively. 
In the first experiment, pre-RHI vs. post-RHI analysis of upper and lower arm lengths

2 revealed a differential effect of the type of stimulation over time (Figure 4; "Time" x "ToS":

$\left.3 F(1,16)=4.5, p=.05, \eta_{\mathrm{p}}{ }^{2}=.22\right)$. Post-hoc tests disclosed that asynchronous condition altered

the perceived lower and upper arm lengths (Post-A vs. Pre-A: $p=.029$ ), while the synchronous condition did not $(p>.05)$. In addition, the perceived lower and upper arm lengths after the asynchronous condition differed from the one measured after the synchronous condition (Post-A vs. Post-S: $p=.034)$. In other words, while the synchronous visuotactile stimulation did not affect the successive performance at the BIT, the asynchronous stimulation resulted in a significant modification of the body metrics of the upper limbs. Note that lower and upper arm segments performed equally but in opposite directions in the statistical tests due to the normalization by total limb length. We did not find any significant interactions or main effects in the perceived length of the lower and upper legs (all $p \mathrm{~s}>.05$ ).

In the second experiment, no interactions or main effects were found for the perceived lower and upper arm lengths (all $p \mathrm{~s}>.05$ ). These results suggest that neither the type of stimulation (observational vs. long-lasting synchronous) nor the time of the stimulation (pre vs. post) seem to affect the body metrics. An interaction between the two factors was found for the leg segments lengths ("Time" $\mathrm{x}$ "ToS": $F(1,16)=4.6, p=.04, \eta_{\mathrm{p}}{ }^{2}=.22$ ). However, post-hoc comparisons did not reveal any significant changes in body metrics for the lower limbs among conditions $(p>.05)$. In other words, just looking at the rubber hand or being subjected to a long (120 s) synchronous visuotactile stimulation did not lead to any difference in body metrics.

We also examined whether the distance between the hand and torso in the BIT shifted after the RHI, since a perceived relocation of the hand has been found in various versions of the RHI paradigm (Tsakiris \& Haggard, 2005; Abdulkarim \& Ehrsson, 2016). Specifically, we calculated the difference between the perceived x position of the hand and the averaged perceived x position of the shoulder and hip on the corresponding side. A value of drift was then 
1 calculated by subtracting this "distance between arm and body" after the RHI from the value

2 before RHI. This was done for each participant, separately for the side that received

3 synchronous stimulation and the side that received asynchronous stimulation. Single t-test

4 revealed drifts not being different from zero for the synchronous $(p=.68)$ or the asynchronous

5 side $(p=.43)$. A paired t-test revealed no difference in drift of the hand between synchronous

6 and asynchronous sides $(p=.88)$.

\section{Discussion}

In the present study we explored whether synchronous and asynchronous visuotactile stimulation of a fake and the real hands result in distortions of the body image, in particular of the perceived size of the stimulated body parts.

We found that synchronous visuotactile stimulation induced a greater sense of body ownership and of referral of touch of the fake hand than asynchronous stimulation did, as expected (Botvinick \& Cohen, 1998). Merely observing the same rubber hand from a firstperson perspective did not induce a strong illusion of ownership of the fake hand. This modulation is unlike full-body illusions, where the mere observation of a collocated virtual body induces the illusion of ownership, with no need for additional multisensory sensorimotor cues, and even despite asynchronous visuotactile stimulation (Maselli \& Slater, 2013).

Our measurements of body metrics using BIT showed that the RHI per se, i.e., executed in a synchronous fashion, did not influence the body metrics, i.e., the perceived length of the arm segments was not altered. Similarly to the synchronous condition, long-lasting synchronous stimulation periods and mere observation of the rubber hand (i.e., without tactile stimulation) did not distort the body metrics. Interestingly, the body metrics represented by the subjects after asynchronous stimulation included a shift in the elbow position. This shift could be due to a primary shortening of the upper arm and with an elongation of the lower arm. We suggest that the primary change is an elongation of the lower arm since the hand position is visually 
anchored by the visible position of the fake hand on the table, but the elbow and shoulder are outside the field of view form a first-person point of view in the position of the experiment.

Regarding the measure used for quantification of the body metrics, the BIT may be more

sensitive to changes in the body image than classical measurements of perceived hand position. Indeed, we did not observe any increase in perceived displacement of the stimulated arm towards the body midline in the synchronous condition compared to the asynchronous condition, unlike some previous studies (Botvinick \& Cohen, 1998). This may be in line with previous studies that have found that proprioceptive drift may not be a determinant indicator of ownership of the fake hand after the RHI (Kammers et al., 2009; Rohde, Di Luca, \& Ernst, 2011; Abdulkarim \& Ehrsson, 2016).

Forty seconds of asynchronous, but not synchronous, stimulation were sufficient to induce a significant modulation in the representation of one's body size, suggesting that the represented body image is not stable and can be modified in a short time by specific patterns of multisensory stimulation. Further, we found no effect on the estimations of metrics of the legs, suggesting that the distortion of body image is not global, but restricted to the body part receiving asynchronous (incongruent) stimulation.

\subsection{Type and Time of Stimulation}

Our results show that asynchronous stimulation can have important effects on the brain processes underlying body image. Nevertheless, it is often used as a control condition in RHI and in virtual hand illusion studies (Slater et al., 2008). It is known that asynchronous visuotactile stimulation does not induce illusion of ownership and that it easily breaks the illusion of ownership induced by synchronous stimulation (Botvinick \& Cohen, 1998; Makin, Holmes, \& Ehrsson, 2008). A recent study suggests that longer stimulation time during visuotactile conflict (i.e., asynchronous visuotactile stimulation) may act primarily on proprioception, without necessarily increasing the sense of body ownership (Rohde et al., 2011). Finally, body ownership over fake (real or virtual) bodies can be induced even in the absence of 
any tactile stimulation (Holmes, Snijders, \& Spence, 2006) if a first-person point of view of the body, a highly effective strategy to induce body ownership (Slater, Spanlang, Sanchez-Vives, \& Blanke, 2010), is provided. This suggests that multisensory synchrony is not the sole driver of body illusions. In this study, longer synchronous stimulation or observation times did not affect body metrics, ruling out an effect due to long-term illusion of ownership. Our results thus confirm that asynchronous stimulation is not strictly "neutral", in the sense of being used as "baseline" or control condition, but rather involves a multisensory conflict that has implications for body representation, specifically for body metrics.

\subsection{The Deafferentation Hypothesis}

Previous studies suggest that synchronous and asynchronous conditions involve different components of the body representation. While in the synchronous condition participants experience a feeling of "loss of hand" or disownership of the real hand, a deafferentation component appears in the asynchronous condition only (Longo et al., 2008; Moseley, 2007). Longo and colleagues related the deafferentation component to the sensation of paraesthesia (tingling sensation, 'pins and needles'), numbness, and the experience of the hand being less vivid than normal. Similar sensations have been documented when visuomotor conflicts are induced by mirrors (McCabe, Haigh, Halligan, \& Blake, 2005). Indeed, actual deafferentation due to local anaesthesia (Gandevia \& Phegan, 1999; Paqueron et al., 2003) or after spinal cord injury (Fuentes et al., 2013) produces a selective increase in the perceived size of a body part. We suggest that the recalibration of the arm segment lengths (i.e., the modulation of the perceived length of the specific body part) induced by asynchronous stimulation may be related to the deafferentation factor in the RHI. This would imply that the modulation of the body image comes through an alteration of the internal representation of the body due to incongruent visuotactile stimulation in the asynchronous condition, which does not occur in the synchronous condition. 


\section{Conclusions}

Our results indicate that the internal representation of the body image is a highly dynamic process. Multisensory stimulation is not only critical for our brain to develop a sense of body ownership with respect to the body, but is also necessary for maintaining a stable and correct internal metric representation of the body. Asynchronous (incongruent) multisensory stimulation, often used as a baseline to measure body ownership illusions, is not a neutral stimulus. Here we demonstrated that asynchronous stimulation induces a recalibration of the metrics of the stimulated limb towards elongation. The present study provides further evidence of how complex and malleable the structure of the internal representation of the body is, highlighting the importance of adequately assessing each component individually.

\section{Acknowledgements}

This research was supported by FP7 EU collaborative projects BEAMING (248620) and VERE (257695). We would like to thank Dr. Cristina Gonzalez-Liencres for her comments on the manuscript.

\section{References}

Abdulkarim, Z., \& Ehrsson, H.H. (2016). No causal link between changes in hand position sense and feeling of limb ownership in the rubber hand illusion. Attention, Perception and Psychophysics, 78, 707-20.

Bookstein, F., Colchester, A., \& Hawkes, D. (1991). Thin-Plate splines and the atlas problem for biomedical images Information Processing in Medical Imaging. Lecture Notes in Computer Science, 511, 326. https://doi.org/10.1007/BFb0033763

Botvinick, M., \& Cohen, J. (1998). Rubber hands "feel" touch that eyes see. Nature, 391(6669), 756. https://doi.org/10.1038/35784

Ehrsson, H. H., Holmes, N. P., \& Passingham, R. E. (2005). Touching a rubber hand: Feeling of body ownership is associated with activity in multisensory brain areas. Journal of Neuroscience, 25, 10564-10573. https://doi.org/10.1523/JNEUROSCI.0800-05.2005

Ehrsson, H. H., Rosen, B., Stockselius, A., Ragno, C., Kohler, P., \& Lundborg, G. (2008). Upper limb amputees can be induced to experience a rubber hand as their own. Brain, 131, 3443-3452. https://doi.org/10.1093/brain/awn297

Flannery, J. C., \& Faria, S. H. (1999). Limb loss: Alterations in body image. Journal of Vascular Nursing, 17, 100-108. https://doi.org/10.1016/S1062-0303(99)90036-5

Fuentes, C. T., Longo, M. R., \& Haggard, P. (2013). Body image distortions in healthy adults. Acta Psychologica, 144, 344-351. https://doi.org/10.1016/j.actpsy.2013.06.012

Gandevia, S. C., \& Phegan, C. M. (1999). Perceptual distortions of the human body image produced by local anaesthesia, pain and cutaneous stimulation. The Journal of Physiology, 514, 609-616. https://doi.org/10.1111/j.1469-7793.1999.609ae.x

Guterstam, A., Petkova, V. I., \& Ehrsson, H. H. (2011). The illusion of owning a third arm. PLoS One, 6, e17208. https://doi.org/10.1371/journal.pone.0017208 
Holmes, N. P., Snijders, H. J., \& Spence, C. (2006). Reaching with alien limbs: Visual exposure to prosthetic hands in a mirror biases proprioception without accompanying illusions of ownership. Perception and Psychophysics, 68, 685-701. https://doi.org/10.3758/BF03208768

Kammers, M. P., Verhagen, L., Dijkerman, H. C., Hogendoorn, H., de Vignemont, F., \& Schutter, D. J. (2009). Is this hand for real? Attenuation of the rubber hand illusion by transcranial magnetic stimulation over the inferior parietal lobule. Journal of Cognitive Neuroscience, 21, 1311-1320. https://doi.org/10.1162/jocn.2009.21095

Kilteni, K., Normand, J. M., Sanchez-Vives, M. V, \& Slater, M. (2012). Extending body space in immersive virtual reality: A very long arm illusion. PLoS One, 7, e40867. https://doi.org/10.1371/journal.pone.0040867

Lloyd, D. M. (2007). Spatial limits on referred touch to an alien limb may reflect boundaries of visuotactile peripersonal space surrounding the hand. Brain and Cognition, 64, 104-109. https://doi.org/10.1016/j.bandc.2006.09.013

Longo, M. R., Kammers, M. P., Gomi, H., Tsakiris, M., \& Haggard, P. (2009). Contraction of body representation induced by proprioceptive conflict. Current Biology, 19, R727-8. https://doi.org/10.1016/j.cub.2009.07.024

Longo, M. R., Schüür, F., Kammers, M. P. M., Tsakiris, M., \& Haggard, P. (2008). What is embodiment? A psychometric approach. Cognition, 107, 978-998. https://doi.org/10.1016/j.cognition.2007.12.004

Makin, T. R., Holmes, N. P., \& Ehrsson, H. H. (2008). On the other hand: Dummy hands and peripersonal space. Behavioural Brain Research, 191, 1-10. https://doi.org/10.1016/j.bbr.2008.02.041

Maselli, A., \& Slater, M. (2013). The building blocks of the full body ownership illusion. Frontiers in Human Neuroscience, 7, 83. https://doi.org/10.3389/fnhum.2013.00083

McCabe, C. S., Haigh, R. C., Halligan, P. W., \& Blake, D. R. (2005). Simulating sensory-motor incongruence in healthy volunteers: Implications for a cortical model of pain. Rheumatology (Oxford), 44, 509-516. https://doi.org/10.1093/rheumatology/keh529

Moseley, G. L. (2007). Using visual illusion to reduce at-level neuropathic pain in paraplegia. Pain, 130, 294-298. https://doi.org/10.1016/j.pain.2007.01.007

Oldfield, R. C. (1971). The assessment and analysis of handedness: The Edinburgh inventory. Neuropsychologia, 9, 97-113. https://doi.org/10.1016/0028-3932(71)90067-4

Paqueron, X., Leguen, M., Rosenthal, D., Coriat, P., Willer, J. C., \& Danziger, N. (2003). The phenomenology of body image distortions induced by regional anaesthesia. Brain, 126, 702-712. https://doi.org/10.1093/brain/awg063

Perez-Marcos, D., Sanchez-Vives, M. V, \& Slater, M. (2011). Is my hand connected to my body? The impact of body continuity and arm alignment on the virtual hand illusion. Cognitive Neurodynamics, 6, 295-305. https://doi.org/10.1007/s11571-011-9178-5

Ramachandran, V. S., \& Hirstein, W. (1998). The perception of phantom limbs. The D. O. Hebb lecture. Brain, 121, 1603-1630.

Rohde, M., Di Luca, M., \& Ernst, M. O. (2009). The time course of proprioceptive drift in the rubber hand illusion. Journal of Vision, 9, 712. https://doi.org/10.1167/9.8.712

Rohde, M., Di Luca, M., \& Ernst, M. O. (2011). The Rubber Hand Illusion: Feeling of ownership and proprioceptive drift do not go hand in hand. PLoS One, 6, e21659. https://doi.org/10.1371/journal.pone.0021659

Schmalzl, L., \& Ehrsson, H. H. (2011). Experimental induction of a perceived "telescoped" limb using a full-body illusion. Frontiers in Human Neuroscience, 5, 34. https://doi.org/10.3389/fnhum.2011.00034

Schmalzl, L., Thomke, E., Ragno, C., Nilseryd, M., Stockselius, A., \& Ehrsson, H. H. (2011). "Pulling telescoped phantoms out of the stump": Manipulating the perceived position of phantom limbs using a full-body illusion. Frontiers in Human Neuroscience, 5, 121. https://doi.org/10.3389/fnhum.2011.00121

Slater, M., Perez-Marcos, D., Ehrsson, H. H., \& Sanchez-Vives, M. V. (2008). Towards a digital body: The virtual arm illusion. Frontiers in Human Neuroscience, 2, 6. https://doi.org/10.3389/neuro.09.006.2008

Slater, M., Spanlang, B., Sanchez-Vives, M. V, \& Blanke, O. (2010). First person experience of body transfer in virtual reality. PLoS One, 5, e10564. https://doi.org/10.1371/journal.pone.0010564 
1 Tsakiris, M., \& Haggard, P. (2005). The rubber hand illusion revisited: Visuotactile integration and selfattribution. Journal of Experimental Psychology: Human Perception and Performance, 31, 8091. https://doi.org/10.1037/0096-1523.31.1.80 


\section{$1 \quad$ Tables}

2

3 Table 1. Means and standard errors for the normalized different body part lengths assessed with 4 the BIT prior to (PRE) and after (POST) asynchronous (A) and synchronous (S) stimulation 5 (Experiment 1).

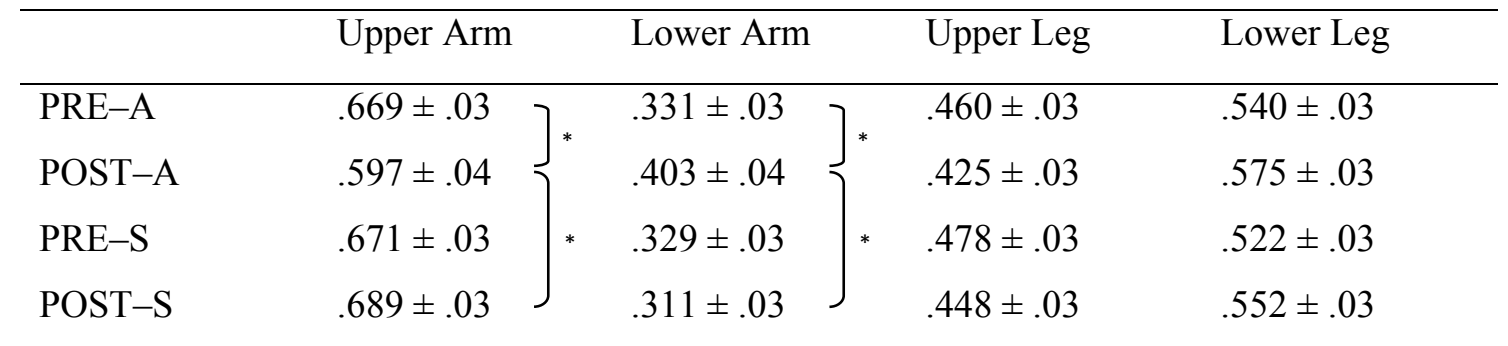

${ }^{*} p<.05$ compared to POST $-\mathrm{A}$ using Newman-Keuls post-hoc test 
2 Table 2. Means and standard errors for the normalized different body parts lengths assessed with 3 the BIT prior to (PRE) and after (POST) the observational (O) and long-lasting synchronous (LS) 4 conditions (Experiment 2).

\begin{tabular}{lllll}
\hline & Upper Arm & Lower Arm & Upper Leg & Lower Leg \\
\hline PRE-O & $.665 \pm .02$ & $.335 \pm .02$ & $.465 \pm .04$ & $.535 \pm .04$ \\
POST-O & $.618 \pm .04$ & $.382 \pm .04$ & $.376 \pm .03$ & $.624 \pm .03$ \\
PRE-LS & $.651 \pm .03$ & $.349 \pm .03$ & $.395 \pm .02$ & $.605 \pm .02$ \\
POST-LS & $.644 \pm .03$ & $.356 \pm .03$ & $.436 \pm .04$ & $.564 \pm .04$ \\
\hline
\end{tabular}

5 


\section{Figure Legends}

2

Figure 1. Experimental RHI setup: All four arms (two rubber, two real) rested palmdown on the desktop, with the rubber arms positioned closer to the body midline. Two partitions prevented direct view of one's own arms. Participants were asked to concentrate on either the left or right rubber hand where tactile stimulation (synchronous or asynchronous, depending on the condition) was delivered to both real and rubber hands of the same side. After $20 \mathrm{~s}$, the complementary stimulation (asynchronous or synchronous) was delivered to the other hand. In the picture, one experimenter strokes both the real and the rubber right hands synchronously.

Figure 2. Mean scores and standard errors for the body ownership and referral of touch questions for the synchronous (S) and asynchronous (A) conditions in the first experiment. $* * * p<.005$, Wilcoxon matched paired-test.

Figure 3. Mean scores and standard errors obtained for the body ownership for the longlasting synchronous (LS) and observational (O) conditions, and for the referral of touch questions (only LS) in the second experiment. ${ }^{* *} p<.005$, Wilcoxon matched paired-test.

Figure 4. Stacked representation of pre-RHI and post-RHI normalized lower and upper arm lengths using the BIT for the synchronous (S) and asynchronous (A) conditions in the first experiment. For each data column, data points represent the relative positions (i.e., normalized with respect to whole arm length) of the hand, the elbow and the shoulder (from bottom to top). $* p<.05$, Newman-Keuls post-hoc test. 